Journal of Innovative Optical Health Sciences

Vol. 10, No. 3 (2017) 1750003 (12 pages)

(c) The Author(s)

DOI: $10.1142 / \mathrm{S} 1793545817500031$

\title{
Fast in vivo bioluminescence tomography using a novel pure optical imaging technique
}

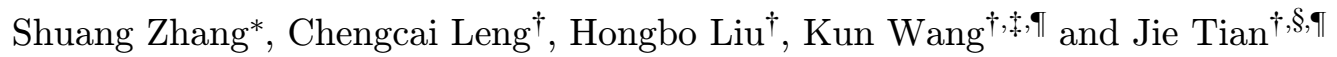 \\ *Sino-Dutch Biomedical and Information Engineering School \\ Northeastern University \\ Shenyang 110819, P. R. China \\ ${ }^{\dagger}$ The Key Laboratory of Molecular Imaging \\ Chinese Academy of Sciences, Zhongguancun East Road \#95, \\ Haidian Dist. Beijing 100190, P. R. China \\ *kun.wang@ia.ac.cn \\ §tian@ieee.org
}

Received 17 August 2016

Accepted 23 November 2016

Published 19 January 2017

\begin{abstract}
Bioluminescence tomography (BLT) is a novel optical molecular imaging technique that advanced the conventional planar bioluminescence imaging (BLI) into a quantifiable three-dimensional (3D) approach in preclinical living animal studies in oncology. In order to solve the inverse problem and reconstruct tumor lesions inside animal body accurately, the prior structural information is commonly obtained from X-ray computed tomography (CT). This strategy requires a complicated hybrid imaging system, extensive post imaging analysis and involvement of ionizing radiation. Moreover, the overall robustness highly depends on the fusion accuracy between the optical and structural information. Here, we present a pure optical bioluminescence tomographic (POBT) system and a novel BLT workflow based on multi-view projection acquisition and 3D surface reconstruction. This method can reconstruct the 3D surface of an imaging subject based on a sparse set of planar white-light and bioluminescent images, so that the prior structural information can be offered for 3D tumor lesion reconstruction without the involvement of CT. The performance of this novel technique was evaluated through the comparison with a conventional dual-modality tomographic (DMT) system and a commercialized optical imaging system (IVIS Spectrum) using three breast cancer xenografts. The results revealed that the new technique offered comparable in vivo tomographic accuracy with the DMT system $(P>0.05)$ in much shorter data analysis time. It also offered significantly better accuracy comparing with the IVIS system $(P<0.04)$ without sacrificing too much time.
\end{abstract}

Keywords: Optical surface reconstruction; bioluminescence tomography reconstruction; optical molecular imaging; light flux reconstruction.

\Corresponding authors.

This is an Open Access article published by World Scientific Publishing Company. It is distributed under the terms of the Creative Commons Attribution 4.0 (CC-BY) License. Further distribution of this work is permitted, provided the original work is properly cited. 


\section{Introduction}

Bioluminescence imaging (BLI) is a powerful tool used in biomedical cancer research. ${ }^{1-3}$ However, it is also known as a qualitative imaging technique as it can only provide planar information over threedimensional (3D) imaging subjects. Recently, bioluminescence tomography (BLT) has been able to provide the quantitative 3D bio-distribution of the bioluminescent photons inside intact living organisms, ${ }^{4-6}$ and thus, it attracted more attention in preclinical small animal studies in oncology..$^{7,8}$ Because of the photo scattering effect in tissues, the 3D tomographic reconstruction of BLI suffers from the challenge of solving the ill-posed inverse problem. ${ }^{9,10}$ A common method proposed to overcome this problem is utilizing prior morphological information of the imaging subject obtained from computed tomography (CT) to optimize the system matrix of BLT and minimize its ill-condition. ${ }^{11}$ Our group has recently advanced this optical-CT dualmodality BLT technique in quantitative imaging of liver $^{9}$ and breast cancer ${ }^{12}$ mouse models. However, this strategy demands a complicated dual-modality tomographic (DMT) system with high standard hardware requirements, extensive and timeconsuming post imaging analysis for morphological segmentation and dual imaging information registration, and the involvement of ionizing radiation. Moreover, the overall robustness of the optical-CT BLT highly depends on the fusion accuracy between the planar bioluminescent images and 3D CT images. This affects the performance of BLT fundamentally, regardless of the efforts in improving the forward model or inverse reconstruction algorithms. ${ }^{8,13}$

To overcome these problems and achieve faster and accurate BLT imaging in vivo, in this study, we introduce a novel BLT technique without the involvement of CT. It employed a pure optical bioluminescence tomographic (POBT) system to acquire both bioluminescent and structural information from a sparse set of planar optical images in multiple angles, a new algorithm based on the Visual Hull method to reconstruct the 3D mouse body surface with the inherent bioluminescent photon flux, ${ }^{14}$ and an algorithm based on the Split Bregman iterative method with $\mathrm{L} 1$ regularization to localize the bioluminescence source in 3D. Three breast cancer xenografts (human breast cancer cell line, MDAMB-231-luc) were used to evaluate the performance of the proposed POBT technique. A conventional
optical-CT DMT system with the same Split Bregman light source reconstruction algorithm and an IVIS Spectrum (PerkinElmer, USA) system with its commercialized BLT algorithm were used to compare with our new technique as references. The results demonstrated that the POBT technique provided promising tumor reconstruction accuracy with much easier hardware setup and much less data analysis time comparing with the reference techniques. We believe the advantages of this new 3D optical imaging technique will further facilitate a wider and easier application of BLT in preclinical cancer researches.

In Sec. 2, the 3D surface reconstruction method and the internal bioluminescence source reconstruction method are introduced in detail. In Sec. 3, the in vivo comparison between the POBT, DMT and IVIS techniques in imaging breast tumor mouse models is demonstrated. The accuracy and time cost for 3D tumor reconstruction are measured, quantitatively, to validate the performance of the proposed system and method. In Sec. 4, the novelty and limitations of this technique are discussed, and the conclusion is summarized in Sec. 5 .

\section{Materials and Methods}

In this section, we describe our technique to reconstruct the location of tumor using POBT and multi-view projection acquisition with $3 \mathrm{D}$ surface reconstruction. Because the oxidation reaction and bioluminescent illumination cannot occur in a death mouse, frozen sections are not used as a standard in this study. Thus, an optical-CT DMT system developed by our group is served as the standard to evaluate the reconstruction reliability of the new method. ${ }^{9}$ Furthermore, a commercialized pure optical IVIS system is also employed for computing the 3D position of tumor as another standard.

The following four steps were implemented for the in vivo pure optical BLT imaging.

1. Animal model preparation: $4-5$ weeks old BALB/c nude mice $(N=3)$ were purchased from the Department of Experimental Animals, Peking University Health Science Center. All animal experiments were performed in accordance with the guidelines of the Institutional Animal Care and Use Committee (IACUC) at Peking University (Permit Number: 2011-0039). The research procedures were approved by IACUC of Peking University and the Key Laboratory of Molecular Imaging, Chinese 
Academy of Sciences (CAS). All surgeries were performed under sodium pentobarbital anesthesia, and all efforts were made to minimize the suffering. The subcutaneous tumor model was established by injecting $1 \times 10^{6}$ cells/ml MDA-MB-231-luc cell suspension $150 \mu \mathrm{L}$ into the right upper flanks of BALB/c nude mice $(N=3)$. After 9 days, the tumor bearing mouse models were employed for the imaging study. Each mouse was intraperitoneally injected with $0.3 \mathrm{~mL}$ anesthetic at a $0.15 \mathrm{~g} / \mathrm{mL}$ concentration. About $10 \mathrm{~min}$ later, $0.3 \mathrm{ml}$ D-Luciferin (Biotium, CA, Fremont, USA)) was intraperitoneally injected.
Then, after $7 \mathrm{~min}$, bioluminescent photons were emitted from the tumor lesion during the oxidation reaction on the condition that luciferase met luciferin.

2. Date acquisition and pre-data analysis: A sparse set of planar white-light images and bioluminescent images was acquired by the POBT and DMT systems (Fig. 1). These images were utilized for white-light and bioluminescent image merging, $3 \mathrm{D}$ mouse surface generation, and 3D volume discretization for the POBT technique. They were also used for the pre-data analysis in the conventional DMT approach together with the acquired CT

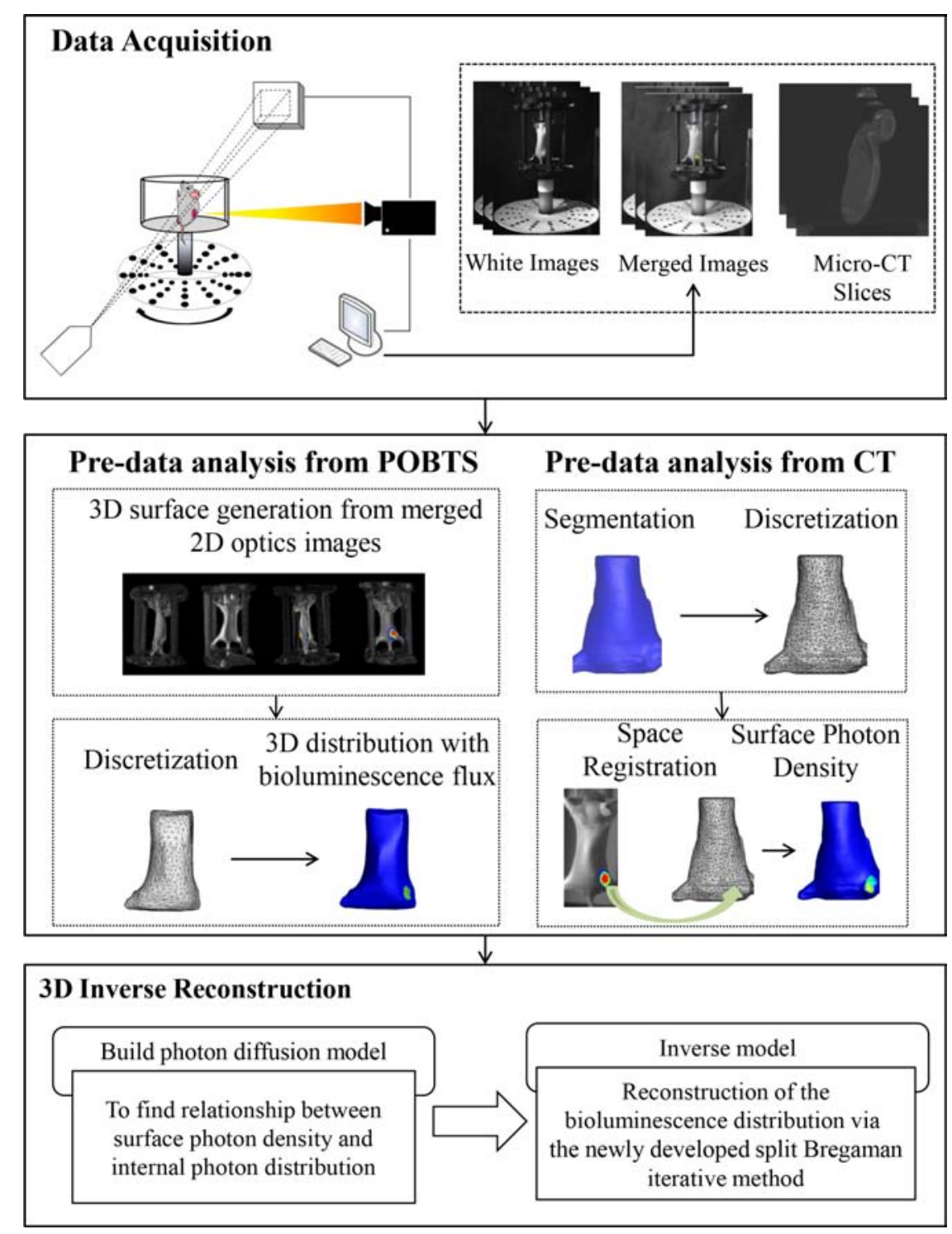

Fig. 1. The overview of the POBT and DMT techniques. Both POBT and DMT systems were intergrated in the same imaging platform and acquried data from the same xenograft. They both shared the same forward imaging model and inverse reconstruction algorithms in the final 3D inverse reconstruction. However, they had different pre-data analysis procedures, and the POBTS simplied this process comparing with the DMT apprach. 
volume data, which includes the CT slice segmentation, 3D mouse surface generation, 3D volume discretization, 2D optical and 3D CT image registration and flux projection (Fig. 1). ${ }^{15}$

3. Three-dimensional reconstruction of the internal photon distribution: The diffusion equation (DE) is utilized to depict how photons travel inside the mouse body, and the finite element method (FEM) is adopted to establish the linear relationship between the photon distribution on the surface and the unknown internal bioluminescence distribution inside the body (Fig. 1). ${ }^{5}$ Here, the unknown bioluminescence intensity distribution, which represents the tumor location and size, was solved using a newly developed algorithm based on the Split Bregman iterative (SBI) method. Both POBT and DMT techniques used the same method in this step.

4. Multi-technique validation: The $3 \mathrm{D}$ rendering of the tumor lesion was visualized by the POBT, DMT and IVIS systems, respectively. The spot (a tetrahedron after discretization) with the highest optical intensity was considered to be the tumor lesion location. The minimum distance between the tumor lesion and the body surface was measured and compared between these three techniques. The total time consumption (pre-data analysis time + inverse reconstruction time) of each technique was also measured and compared. Statistical comparisons were made using Student's T-test. $P$ values less than 0.05 were considered to indicate significance. Average and standard deviations were calculated for this experiment performed in triplicate.

\subsection{The imaging system description}

The schematic illustration of the imaging system is given by Fig. 2. The system was built in a dark room with radiation-proof shielding. Both POBT and DMT systems were integrated on the same imaging platform.

The POBT system consisted of an ultrasensitive cooled EM-CCD camera (Andor's iXon3888, UK, pixel size: $13 \mu \mathrm{m} \times 13 \mu \mathrm{m}$ ), a rotating stage with a mouse holding shelf. Underneath the rotating stage, there was a white calibration mat with a series of black dots as special markers (Fig. 2(a)). It provided marking information to help the later $3 \mathrm{D}$ mouse surface generation. In our experiment, a sparse set of planar white-light and bioluminescent images of the mouse was acquired by the Andor

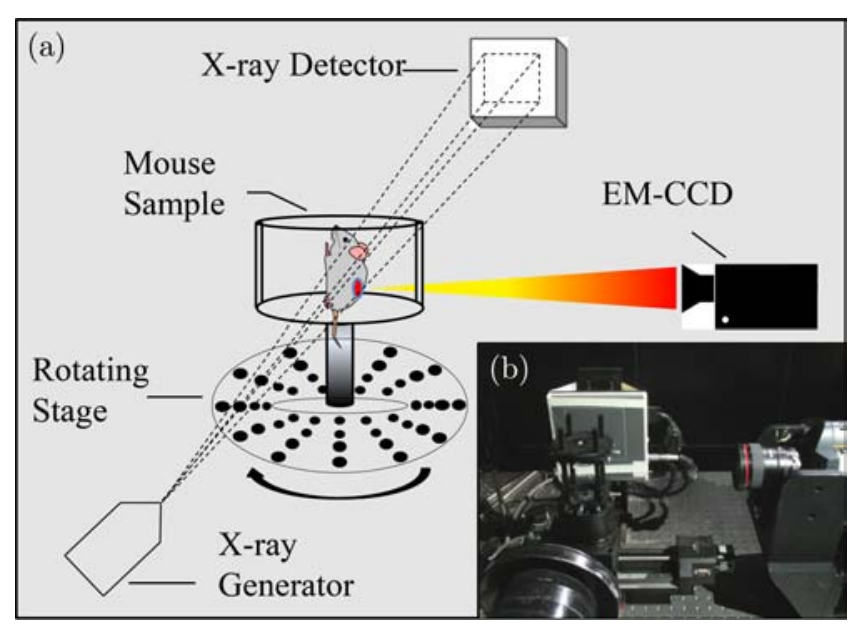

Fig. 2. The POBT and DMT dual BLT imaging systems. (a) The schematic illustration of the POBT and DMT systems. Both systems shared the same rotating stage, but the underneath calibration mat was only used for the POBT technique. (b) A picture of the system set-up, including the cooled EMCCD, X-ray generator, X-ray detector, and Rotating stage.

EM-CCD, and they can be easily merged because of their sharing of the same imaging acquisition angle. Then, the 3D mouse surface was generated from these merged optical planar images using the "Visual Hull" approach. ${ }^{16}$ Furthermore, the planar texture information was also mapped into the generated 3D surface structure. Therefore, both the morphological information of the mouse body and the bioluminescent flux were obtained in 3D using this easily implemented hardware system.

Besides the optical components and the rotating stage, the DMT system also employed the X-ray generator and detector for CT image acquisition. Different from the POBT technique, it does not need the calibration mat and the $3 \mathrm{D}$ mouse surface generation algorithm. However, it needs sufficient CT image segmentation and optical-CT image registration algorithms implemented inside the system to obtain the morphological information of the mouse body mapped with the bioluminescent flux. Moreover, the DMT system involved significant amount of ionizing radiation.

To further validate the performance of our house-developed systems, we also employed the IVIS Spectrum system to achieve 3D BLT. It is a widely used commercialized pure optical imaging system with tomographic imaging abilities. Since its hardware setup and software are confidential for public customers, we only used its BLT imaging results for multi-systems comparison in this study. 


\subsection{D mouse surface generation}

To summarize, surface mesh generation and texture map generation were the main two steps of the approach for 3D surface reconstruction. First, the surface model was generated using a "shape from silhouettes" approach, from which the exact "Visual Hull" was quickly calculated through a novel batch method. ${ }^{16}$ Then, we applied a robust and efficient technique for blending the image data to generate a consistently textured model without the associated loss of texture details.

Generating 3D models from photographs has received much interest in the Computer Vision, academically and commercially. ${ }^{17}$ There are many possible techniques in recent years for computing the 3D morphology of an imaging subject. ${ }^{18,19} \mathrm{In}$ our work, we aimed to preserve sufficient image details to build very high quality $3 \mathrm{D}$ shape for accurate bioluminescent inverse reconstruction without sacrificing too much computational time. Therefore, we applied one of the classic silhouette methods named visual hull, which uses the maximum volume to reproduce all silhouettes of an object. ${ }^{16}$ This method has excellent robustness and speed in generating 3D models of objects with simple shapes. ${ }^{20}$

We used a volumetric sampling approach to generate a mesh representation of the visual hull. First, an initial volume containing the whole mouse body needed to be defined. Then, it was divided into a union of a set of small cubes. Then, we recursively kept cubes in front of all camera projection angles and discarded those that lie totally behind any camera projection angle. The cubes that were partially in front of all the camera projection angles were subdivided into smaller sizes, and then we repeated this evaluation until all the cubes were in front of all angles or a minimum size was reached. In order to speed up the calculation, we stored these cubes in an octree structure. Nodes in the octree were projected into the silhouette images to determine if they were fully inside or outside the visual hull. In this way, a volumetric representation of the visual hull was generated. This procedure was computationally expensive and required a large memory overhead. To avoid oversampling, the model generally required an error-parameter $E$ to manage the size of the system memory. $E$ is the error-parameter of silhouette from all views.

$$
E=\frac{1}{N} \sum_{K=1}^{N} \Phi_{K}^{J}-\Phi_{K}^{I},
$$

where $N$ is the number of white-light images. $\Phi_{K}^{I}$ is the binary profile of the view $k(k=1, \ldots, N)$ obtained by the camera from different projection angles, and $\Phi_{K}^{J}$ is the back-projected binary silhouette of the mouse. It stands for the total number of average projection error between pixels of the two binary silhouettes in each view $k$. Then, we set an average error parameter $Q$. If $E>Q$, cubes were further divided until $E<=Q$. When $E<=Q$, we ended the subdivision, and the $3 \mathrm{D}$ mouse morphology was generated.

Since, for each camera projection angle, a bioluminescence image and a white-light image were both acquired by our POBT system, the 2D merged images from all angles can be obtained easily. Then, we mapped all merged images into the surface of the new generated 3D mouse model. Because this model was generated from these $2 \mathrm{D}$ images, this texture mapping procedure ensured a very accurate and robust registration of the bioluminescent flux into the 3D mouse model, which is critical for the accurate reconstruction of the light source (tumor lesion) under the mouse skin in the next step. After the texture mapping, the 3D mouse model was triangulated using the Exact Visual Hull Marching Cubes method. ${ }^{21}$

\subsection{Light source reconstruction in $B L T$}

For photon propagation in biological tissues with the optical properties of high scattering and low absorption, the diffusion equation is usually adopted to describe the propagation of light through the mouse. ${ }^{4}$ Normally, the bioluminescent light is considered to be a diffusion-dominant transport process in biological tissues for its red weighted spectrum. ${ }^{10}$

The diffusion equation is the first-order spherical harmonics approximation for the radiative transfer equation, ${ }^{22}$ which can be written as Eq. (2) in the steady-state domain.

$$
-\nabla \cdot[D(r) \nabla \Phi(r)]+\mu_{a}(r) \Phi(r)=S(r), r \in \Omega,
$$

where $D$ is the optical diffusion coefficient, and

$$
D=\frac{1}{3\left[\mu_{a}+(1-g) \mu_{s}\right]} .
$$


$\mu_{a}$ is the absorption coefficient, $g$ is the anisotropy parameter, $\mu_{s}$ is the scattering coefficient, $r$ is the position vector, $\Phi$ is the photon flux density, $S$ is the signal source distribution and $\Omega$ is the region of biological tissues.

The diffusion equation is accompanied by the Robin boundary condition,,$^{9,23}$ which is given by

$$
\Phi(r)+2 A\left(r ; n, n^{\prime}\right) D(r)[\nu(r) \cdot \nabla \Phi(r)]=0, r \in \partial \Omega
$$

After applying the process of discretization by finite element formulation to the photon diffusion model, we established the linear relationship between the measured outgoing photon distribution on the surface and the unknown internal photon distribution via replacing the variables with the matrix-vector forms: ${ }^{23}$

$$
A S=\tilde{\Phi},
$$

where $A^{m \times n}(m \leq n)$ is the system matrix standing for the optical properties of the heterogeneous mouse model and $\tilde{\Phi}$ is the surface photon flux density acquired by the ultrasensitive CCD camera of the system. Therefore, the ultimate aim of BLT was to recover the bioluminescent source distribution $S$ in the above linear equation by an effective optimization strategy. ${ }^{5}$

Due to insufficient measurement and the high diffusive nature of the photon propagation in tissues, this problem is ill-posed. In order to obtain a unique solution, the regularization was used to determine source power density by minimizing the following objective function:

$$
\min _{S} \frac{\mu}{2}\|A S-\tilde{\Phi}\|_{2}^{2}+\|S\|_{1},
$$

where $\mu$ is positive regularization parameter balancing the data fidelity and the regularization term. Then, Eq. (6) can be rewritten as Eq. (7) with intermediate variable to decoupling,

$$
\min _{S, d}\|d\|_{1}+\frac{\mu}{2}\|A S-\tilde{\Phi}\|_{2}^{2} \text { s.t. } d=S .
$$

Then, we transform the Eq. (7) to Eq. (8) by using the euler-lagrange operator,

$$
\min _{S, d}\|d\|_{1}+\frac{\mu}{2}\|A S-\tilde{\Phi}\|_{2}^{2}+\frac{\lambda}{2}\|d-S\|_{2}^{2} .
$$

In order to optimize Eq. (8), we exploited an effective Split Bregman algorithm with $\mathrm{L}_{1}$-Norm to solve the optimization problem. ${ }^{24,25}$

According to the Bregman iterative framework, the two simple sub-problems were presented as follows:

$$
\begin{aligned}
\left(S^{k+1}, d^{k+1}\right)= & \min _{S, d}\|d\|_{1}+\frac{\mu}{2}\|A S-\tilde{\Phi}\|_{2}^{2} \\
& +\frac{\lambda}{2}\left\|d-S-b^{k}\right\|_{2}^{2} \\
b^{k+1}= & b^{k}+S^{k+1}-d^{k+1} .
\end{aligned}
$$

Equations (9) and (10) were then solved by three simple iterative steps:

$$
\begin{gathered}
S^{k+1}=\arg \min _{S} \frac{\mu}{2}\|A S-\tilde{\Phi}\|_{2}^{2} \\
+\frac{\lambda}{2}\left\|d^{k}-S-b^{k}\right\|_{2}^{2} \\
d^{k+1}=\arg \min _{d}\|d\|_{1}+\frac{\lambda}{2}\left\|d-S^{k+1}-b^{k}\right\|_{2}^{2} \\
b^{k+1}=b^{k}+S^{k+1}-d^{k+1},
\end{gathered}
$$

where $\mu$ is regularization parameter, $\lambda$ is the Spilt Bregman iterative algorithm parameter. The regularization parameter $\lambda=10^{n}$ was selected with different orders of magnitude, with $n$ ranging from 1 to 10 ; as for the $\mu=10^{n}$, with $n$ ranging from -10 to 0. Then, Eq. (11) can be rewritten as Eq. (14) with intermediate variable to decoupling,

$S^{k+1}=\left(\mu A^{T} A+\lambda I\right)^{-1}\left(\mu A^{T} \tilde{\Phi}+\lambda\left(d^{k}-b^{k}\right)\right)$.

The optimal value of $d$ can be computed using adjusted shrinkage operators for Eq. (12). Thus,

$$
\begin{aligned}
d^{k+1}= & \operatorname{sign}\left(S^{k+1}+b^{k}\right) \\
& \times \max \left(0,\left|S^{k+1}+b^{k}\right|-\frac{1}{\lambda}\right) .
\end{aligned}
$$

The main outline of the Spilt Bregman algorithm with $l_{1}$-Norm is given in Algorithm 1. For the stopping condition, we choose $\left\|S^{k+1}-S^{k}\right\| /\left\|S^{k}\right\|>\varepsilon=$ $1.0 \times 10^{-3}$.

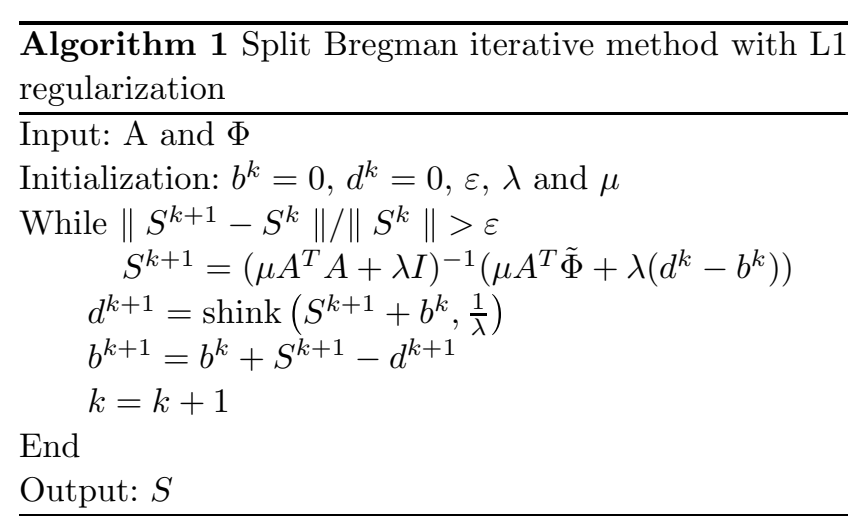




\section{Experimental Results}

To verify the performance of our POBT system with multi-view projection acquisition and $3 \mathrm{D}$ surface reconstruction, as well as the Split Bregman algorithm for fast BLT, we conducted in vivo experiments with multi-system comparisons. Three xenografts were employed to perform the BLT using our POBT system, the conventional DMT system, and the IVIS Imaging System Spectrum (PerkinElmer, USA).

\subsection{Data acquisition and image analysis}

During the imaging acquisition, white-light and bioluminescent light images were captured from 15 projection angles with $24^{\circ}$ interval in our POBT system (Fig. 3(a)). The data acquisition time plus the $3 \mathrm{D}$ surface reconstruction time for the entire procedure was less than $15 \mathrm{~min}$. Figures 3(b) and 3(c) show the details of two examples at the view of $0^{\circ}$ and $48^{\circ}$. Each case illustrates the planar white light image, the $2 \mathrm{D}$ merged image and the corresponding $3 \mathrm{D}$ surface reconstruction. The tumor lesion was indicated by the red circle and arrow. The bioluminescent light emitted from the tumor was captured. The 3D surface reconstruction demonstrated a good morphological structure of the mouse body with rich details. Although there was some loss of details of the mouse head and upper limbs (Figs. 3(b) and 3(c)), which was due to the relatively complicated structure around this part of the body, the structure of the mouse body with the tumor lesion was successfully reconstructed. This was crucial for the later reconstruction of the bioluminescent light source distribution, as it offered the prior morphological information of the xenografts for solving the inverse problem in BLT without using the X-ray CT images.

Since the performance of the optical/micro-CT DMT system developed by our laboratory was thoroughly investigated in previous studies, ${ }^{9}$ it was employed to validate the performance of the new POBT system. In DMT, both optical and X-ray CT images of a same imaging mouse were acquired. It normally took $20-25 \mathrm{~min}$ to finish the dual modality data acquisition, and the morphological structure of the mouse body surface was obtained by the semi-automatic segmentation using the MITK (http://mitk.org/wiki/MIT) during the offline imaging analysis. The optical images were

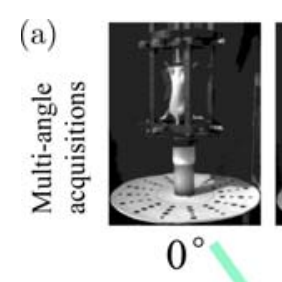

(b)

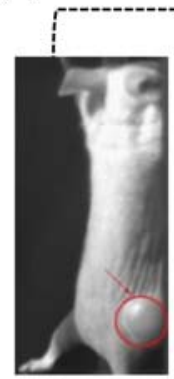

White light

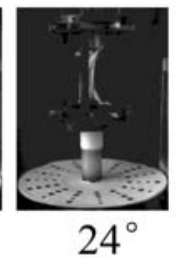

$24^{\circ}$

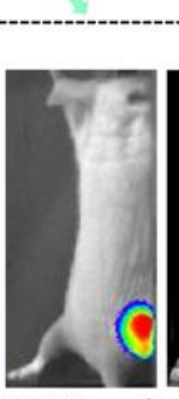

2D Merged
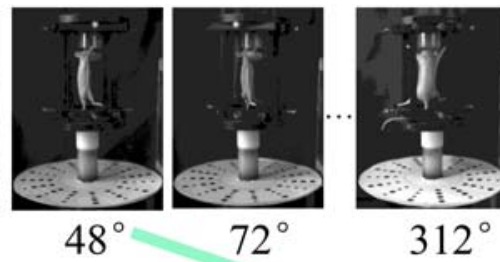

$312^{\circ}$

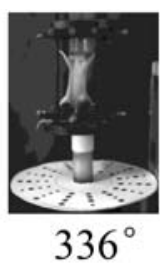

(c)

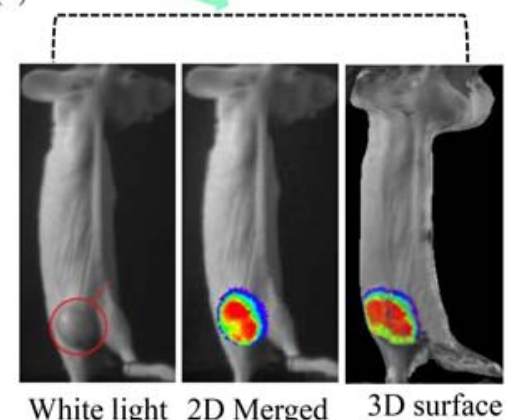

reconstruction

Fig. 3. Results of the multi-view imaging acquisition and the 3D surface generation. (a) The sequential 2D white-light images of a tumor bearing mouse model was acquired in different projection angles. (b) The examples of the planar white-light image, the planar bioluminescent and white-light merged image, and the 3D surface reconstruction are demonstrated from left to right. The red circle and arrow indicate the location of the tumor. (c) Another example of the same arrangement as (b) at $48^{\circ}$. The $3 \mathrm{D}$ surface reconstruction in both (b) and (c) shows a good correlation in shapes with the corresponding 2D merged images. 


\section{S. Zhang et al.}

mapped onto the surface of the volumetric mesh in terms of space and energy. ${ }^{9}$ Then the homogeneous mouse model was discretized into a volumetric mesh containing 4,513 modes and 21,586 tetrahedral elements. The total time of both data acquisition and image analysis was $2 \mathrm{~h}$ for each mouse. Finally, the reconstruction based on the Split Bregman iterative algorithm was performed to localize the tumor.

\subsection{Reconstruction results of the xenografts}

In this section, all BLT reconstruction results given by the POBT, DMT and IVIS systems were represented to evaluate the accuracy and speed of the proposed technique. To make the comparison fair, all the computational processes were conducted on a personal computer with $3.09 \mathrm{GHz}$ Intel Core i5 CPU and 3.46 GB RAM.

Both POBT and DMT techniques achieved successful bioluminescent light source reconstruction of all three xenografts. The 3D mouse body surfaces with bioluminescent flux obtained by these two systems are shown in Fig. 4(a), respectively, with the same color scale.

The corresponding 3D light source reconstructions of the BLT given by the two techniques are shown in Fig. 4(b). These results demonstrated that both techniques provided similar $3 \mathrm{D}$ reconstruction

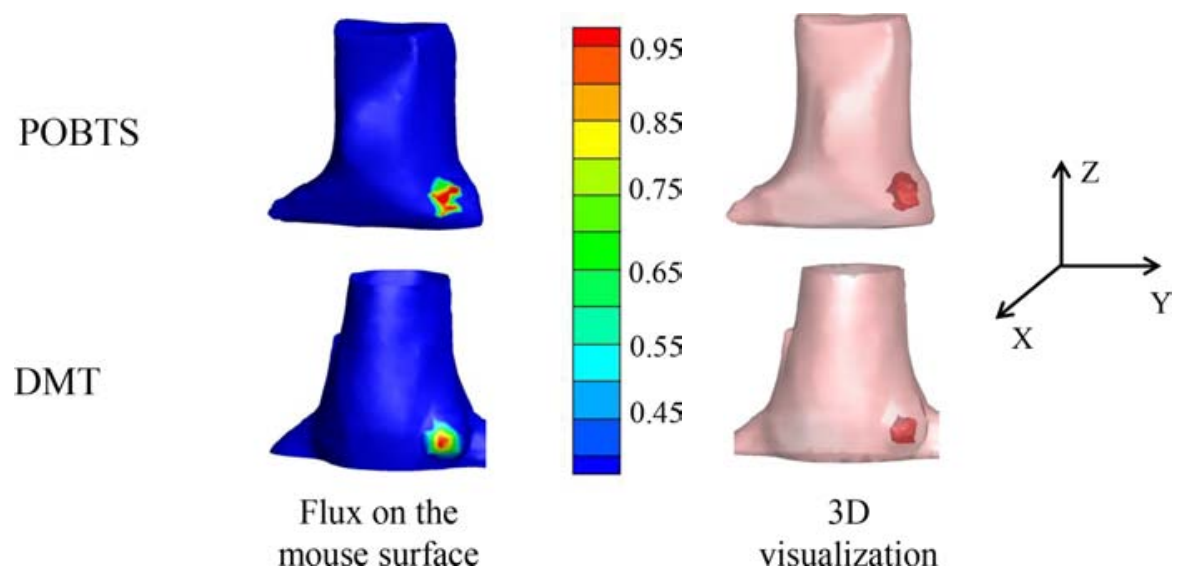

(a)

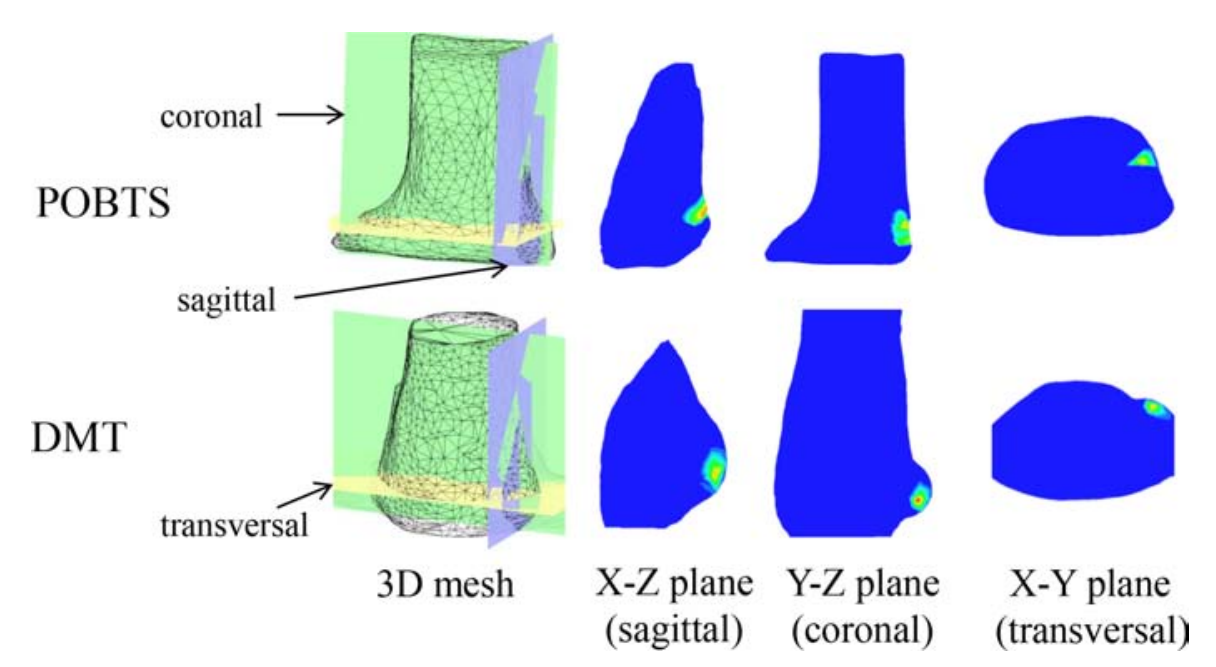

(c)

Fig. 4. The reconstruction results of the POBT and DMT techniques. (a) The 3D mouse body surface with bioluminescent flux given by the POBT (top) and DMT (bottom) techniques, respectively. (b) The corresponding 3D light source reconstructions of the BLT given by the POBT (top) and DMT (bottom) techniques, respectively. (c) The coronal, sagittal and transversal slices were extracted from the POBT and DMT 3D BLT datasets, within the same X-Y-Z coordinate. 
results of the bioluminescent light source inside the mouse body in the case when $\lambda=10$ and $\mu=1$. Within the same X-Y-Z coordinate, the coronal, sagittal and transversal slices were extracted from the two 3D BLT datasets and shown in Fig. 4(c). These results revealed that although there was a bit difference between the 3D configurations of the mouse body obtained from the POBT and CT volume data, the spatial positions of the light source reconstructed by the two techniques matched each other.

So far, there are some commercial devices to implement the functionality of the tumor location reconstruction. IVIS Imaging System Spectrum is one of widespread application devices, ${ }^{26,27}$ and it is also a pure optical 3D imaging system. To further investigate the performance of our POBT technique, we adopted this commercial system to acquire the fluorescence and white light images, and followed the guideline of the IVIS Living Imaging 3.0 software (PerkinElmer, USA) to achieve 3D light source reconstruction. Figure 5(a) shows the 3D tumor lesion reconstruction of the IVIS Imaging System Spectrum. The true tumor lesion was indicated by the red circle and arrow. Within the same $\mathrm{X}-\mathrm{Y}-\mathrm{Z}$ coordinate, the coronal, sagittal and transversal slices were extracted from IVIS Imaging System Spectrum datasets and shown in Figs. 5(b) and 5(c). The red arrow (Fig. 5(c), the bottom slice) was the minimum distance between the reconstructed tumor lesion center and the true tumor lesion surface.

\subsection{The quantitative comparison between PBOT, DMT and IVIS technique}

Figures $6(\mathrm{a})-6(\mathrm{c})$ show transversal images of the same xenograft with the tumor lesion indication obtained from the IVIS Imaging System Spectrum, the POBT system and the DMT system, respectively. Figure $6(\mathrm{~d})$ shows the normalized intensity profiles of the three techniques along the corresponding sample lines (Figs. 6(a)-6(c), red dotted lines). The intensities are plotted in the function of the actual distance in the transversal plane of the xenograft.

The transversal images are shown with the same orientation to facilitate comparisons, although an accurate co-registration is not possible due to inevitable body deformations during image acquisition in the different hardware systems. The reconstructed tumor location of the POBT matched that of the DMT technique (Figs. 6(b) and 6(c)). However, there was a distinct difference in tumor locations between the IVIS and the POBT or DMT technique (Figs. 6(a)-6(c)). The pixel intensity profiles in Fig. 6(d) further quantified this

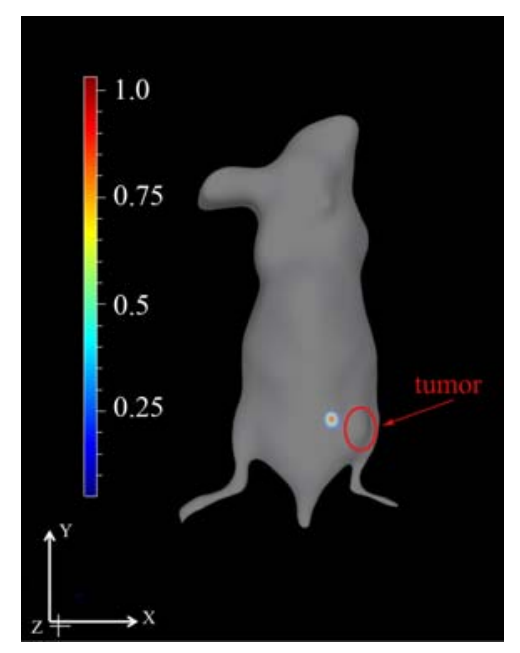

(a)

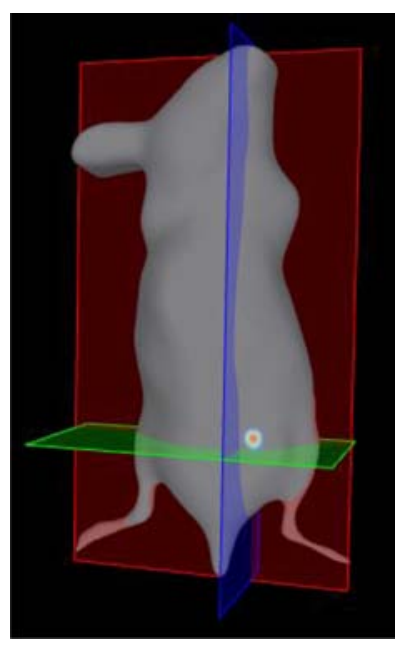

(b)

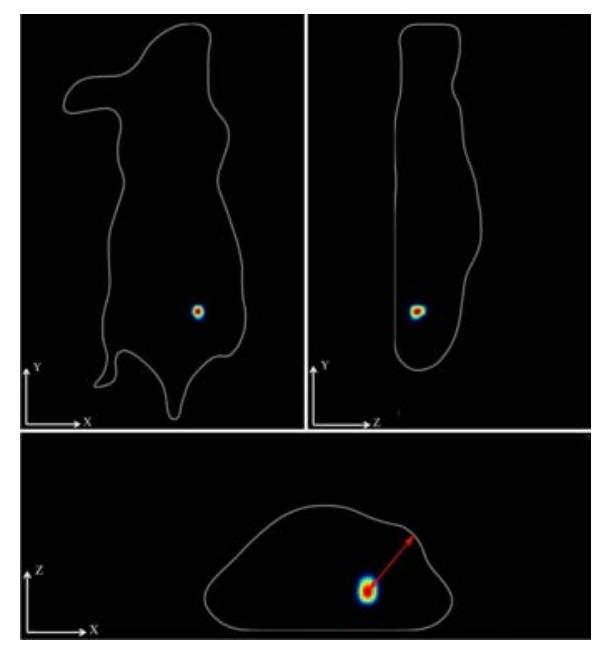

(c)

Fig. 5. Reconstruction results of the IVIS Imaging System Spectrum. (a) The 3D tumor lesion reconstruction of the IVIS Imaging System Spectrum. The true tumor location is indicated by the red circle and arrow. The reconstructed light source is inside the mouse body but not inside the true tumor lesion. (b)-(c) The coronal, sagittal and transversal slices were extracted from the IVIS Imaging System Spectrum dataset, within the same X-Y-Z coordinate. The red arrow was the minimum distance between the reconstructed tumor center and the true tumor surface on the transversal slice. 


\section{S. Zhang et al.}

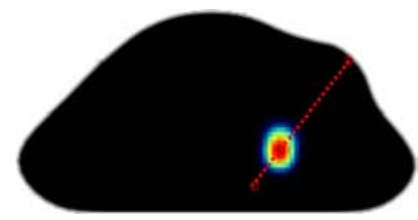

(a)

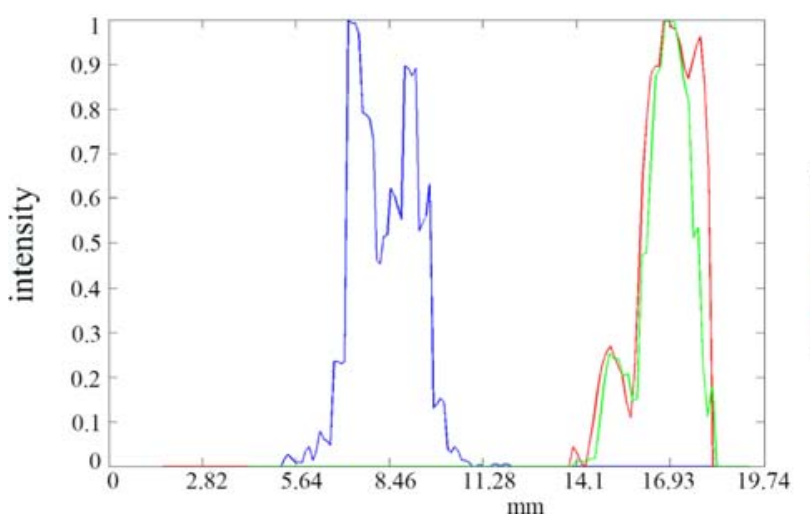

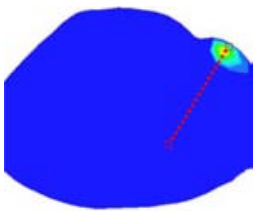

(c)

(d)

Fig. 6. Intensity profiles of IVIS, POBT and DMT techniques. (a)-(c) Transversal planes of the same xenograft with the tumor lesion indication reconstructed by IVIS, POBT and DMT techniques, respectively. (d) The normalized intensity profiles of the IVIS image (blue curve), the POBT image (red curve) and the DMT image (green curve) along the red dotted lines in (a)-(c). The $x$-axis in (d) denotes the actual distance in millimeters along these red dotted lines.

difference. The peaks of blue curve (IVIS) were far away from the peaks of green (DMT) and red (POBT) curves, while the peaks of the latter two curves overlapped each other. This revealed that the center location of the tumor reconstructed by the IVIS technique was deep inside the mouse body rather than inside the true tumor lesion, which did not match the other two techniques. The average distance from the reconstructed tumor center to the tumor lesion surface was POBT: $2.81 \pm 2.37 \mathrm{~mm}, \quad$ DMT: $2.76 \pm 2.13 \mathrm{~mm}$, and IVIS: $7.07 \pm 1.36 \mathrm{~mm}$. There was no significant difference between POBT and DMT $(P=0.28)$, but there was significant different between IVIS and DMT $(P=0.04)$.

To examine the computational efficiency, IVIS Imaging System Spectrum and DMT system have been applied once again to reconstruct the same datasets in contrast to the proposed method. The reconstruction time of the three different techniques has been compared in Table 1. The DMT technique required more than $2 \mathrm{~h}$ to pre-process the data for reconstruction, while POBT considerably enhanced the efficiency up to $0.25 \mathrm{~h}$. The IVIS technique was the fastest option for3D BLT. However, its accuracy was not reliable.
Table 1. Reconstruction efficiency of DMT, POBT and IVIS.

\begin{tabular}{lccc}
\hline & $\begin{array}{c}\text { Pre-data } \\
\text { analysis time }\end{array}$ & Reconstruction time & Total time \\
\hline DMT & $2 \mathrm{~h}$ & $5.31 \mathrm{~s}$ & $2 \mathrm{~h}+5.31 \mathrm{~s}$ \\
POBT & $0.252 \mathrm{~h}$ & $5.31 \mathrm{~s}$ & $0.25 \mathrm{~h}+5.31 \mathrm{~s}$ \\
IVIS & $0.05 \mathrm{~h}$ & $2 \mathrm{~s}$ & $0.05 \mathrm{~h}+2 \mathrm{~s}$ \\
\hline
\end{tabular}

\section{Discussion and Conclusion}

BLI is a powerful preclinical research tool for various disease studies in small animal models. In this paper, we have developed an in vivo image reconstruction technique for $\mathrm{BLT}$ that recovers the $3 \mathrm{D}$ reporter probe distribution inside small animals. This approach used a house-made POBT hardware system for multi-view projection acquisition, including white-light and bioluminescent light, and newly developed algorithms for 3D surface reconstruction (the visual hull method) and inverse light source reconstruction (the Split Bregman iterative method). Therefore, it avoided the involvement of ionizing radiation from X-ray $\mathrm{CT}$, the complicated operation protocol for dual modality data acquisition, and the expensive hardware setup in 
comparison with conventional optical-CT DMT approach. ${ }^{28,29}$

Besides these advantages, the in vivo imaging performance of xenografts in this novel technique was validated through a thorough comparison with the conventional DMT technique and the commercialized IVIS technique. The results demonstrated several unique features of the POBT approach. It can provide relatively accurate tumor localization with less time and labor consumption. Although the $3 \mathrm{D}$ mouse surface reconstruction did not give exactly the same mouse body morphology as the CT did (Fig. 4), it still provided sufficient geometric structure of imaging subjects as the prior information for the later inverse reconstruction of tumor lesions. Because both prior structural and bioluminescence molecular information were obtained from the same CCD camera and the same projection angles, the new method did not need the optical-CT imaging registration or bioluminescent flux mapping on CT volumetric images. Therefore, it omitted the process of CT image segmentation, which frequently required manual correction, and eliminated the inaccuracy of dual modality imaging registration. All of these considerably decreased the image acquisition time and the imaging analysis time for the BLT reconstruction of each imaging subject with insignificant sacrifice of the imaging accuracy $(P=0.28)$ in comparison with the conventional DMT technique, when the same inverse reconstruction algorithm was applied.

The IVIS technique also can offer a pure optical BLT imaging solution without the involvement of CT. It was the fastest one among the three 3D imaging approaches (Table 1). However, the reconstructed location of the bioluminescent source was significantly different with the reference DMT method $(P=0.04)$. For all three subcutaneous tumor bearing mouse models, the IVIS technique indicated that the tumor lesion was deep inside the mouse body (Fig. 6), which was apparently not reliable. Therefore, the novel POBT technique combined the advantages of the DMT and the IVIS methods in order to achieve a new balance between the imaging accuracy and the imaging speed for in vivo BLT. It demonstrated a great potential for minimizing the time cost of $3 \mathrm{D}$ quantitative BLI from hours to minutes without significantly reducing the accuracy. This is particularly beneficial in in vivo mouse studies with large population.
There were two major limitations of the current POBT technique. One is that the 3D mouse surface reconstruction is not suitable for geometrically complicated parts of a mouse body, such as the head and limbs, but this can be overcome by the continuous improvement of the surface reconstruction algorithm. The other is the inaccuracy of the inverse light source reconstruction caused by the homogeneous $^{30}$ assumption of a mouse body. This can be reduced in the conventional DMT technique by adding more optical parameters in different organs and tissues, but this heterogeneous ${ }^{9}$ approach is inapplicable in the POBT technique. ${ }^{31}$

In conclusion, the novel POBT technique was able to provide promising tumor reconstruction accuracy with much easier hardware setup (without any ironizing radiation) and much less data analysis time. It showed great potential for extending the application of quantitative in vivo BLT imaging in preclinical studies with large population.

\section{Acknowledgments}

This work is supported by the National Basic Research Program of China (973 Program) under Grant No. 2015CB755500, the National Natural Science Foundation of China under Grant No. 81227901, 61231004, 81527805 and 61401462, and the Scientific Research and Equipment Development Project of the Chinese Academy of Sciences under Grant No. YZ201359. The Key Research Program of the Chinese Academy of Sciences under Grant No. KGZD-EW-T03, the Chinese Academy of Sciences Fellowship for Young International Scientists under Grant No. 2013Y1GA0004 and the Project funded by China Postdoctoral Science Foundation under Grant Nos. 2014M550881, 2015T80155.

\section{References}

1. T. Maldiney, A. Bessiere, J. Seguin et al., "The in vivo activation of persistent nanophosphors for optical imaging of vascularization, tumours and grafted cells," Nat. Mater. 13(4): 418-426 (2014).

2. J. T. Au, L. Gonzalez, C. H. Chen, I. Serganova, Y. Fong, "Bioluminescence imaging serves as a dynamic marker for guiding and assessing thermal treatment of cancer in a preclinical model," Ann. Surg. Oncol. 19(9): 3116-3122 (2012).

3. R. Weissleder, M. J. Pittet, "Imaging in the era of molecular oncology," Nature 452(7187): 580-589 (2008). 
4. C. Qin, J. Feng, S. Zhu, X. Ma, J. Zhong, P. Wu, Z. Jin, J. Tian, "Recent advances in bioluminescence tomography: Methodology and system as well as application," Laser Photonics Rev. 8(1): 94-114 (2014).

5. C. Leng, J. Tian, "Mathematical method in optical molecular imaging," Sci. China-Inf. Sci. 58(3): 1-13 (2015).

6. C. Darne, Y. Lu, E. M. Sevick-Muraca, "Small animal fluorescence and bioluminescence tomography: A review of approaches, algorithms and technology update," Phys. Med. Biol. 59(1): 1-64 (2014).

7. K. Liu, Y. Lu, J. Tian, C. Qin, X. Yang, S. Zhu, X. Yang, Q. Gao, D. Han, "Evaluation of the simplified spherical harmonics approximation in bioluminescence tomography through heterogeneous mouse models," Opt. Express. 18(20): 20988-21002 (2010).

8. Y. Lv, J. Tian, W. Cong, G. Wang, J. Luo, W. Yang, H. Li, "A multilevel adaptive finite element algorithm for bioluminescence tomography," Opt. Express. 14(18): 8211-8223 (2006).

9. P. Wu, K. Liu, Q. Zhang, Z. Xue, Y. Li, N. Ning, X. Yang, X. Li, J. Tian, "Detection of mouse liver cancer via a parallel iterative shrinkage method in hybrid optical/microcomputed tomography imaging," J. Biomed. Opt. 17(12): 126012 (2012).

10. W. Ping, H. Yifang, W. Kun, T. Jie, "Bioluminescence tomography by an iterative reweighted 12-norm optimization," IEEE Trans. Biomed. Eng. 61(1): 189-196 (2014).

11. C. C. Kun Wang, Z. H. Hu et al., "Optical molecular imaging frontiers in oncology: The pursuit of accuracy and sensitivity," Engineering 1(3): 309-323 (2015).

12. Q. Zhang, Y. Du, Z. Xue, C. Chi, X. Jia, J. Tian, "Comprehensive evaluation of the anti-angiogenic and anti-neoplastic effects of Endostar on liver cancer through optical molecular imaging," PloS one $\mathbf{9}(1)$ : (2014).

13. X. J. Gu, Q. H. Zhang, L. Larcom, H. B. Jiang, "Three-dimensional bioluminescence tomography with model-based reconstruction," Opt. Express. 12(17): 3996-4000 (2004).

14. S. Zhang, K. Wang, C. Leng, K. Deng, Y. Hu, J. Tian, "In vivo bioluminescence tomography based on multi-view projection and 3D surface reconstruction," Proc. SPIE 9328: 93280S (2015).

15. R. A. Weersink, S. Ansell, A. Wang, G. Wilson, D. Shah, P. E. Lindsay, D. A. Jaffray, "Integration of optical imaging with a small animal irradiator," Med. Phys. 41(10): 102701 (2014).

16. A. Laurentini, "The visual hull concept for silhouette-based image understanding," IEEE Trans. Pattern Anal. Mach. Intell. 16(2): 150-162 (1994).
17. M. I. Restrepo, A. O. Ulusoy, J. L. Mundy, "Evaluation of feature-based 3-D registration of probabilistic volumetric scenes," ISPRS-J. Photogramm. Remote Sens. 98: 1-18 (2014).

18. K. N. Kutulakos, S. M. Seitz, "A theory of shape by space carving," Int. J. Comput. Vis. 38(3): 199-218 (2000).

19. H-x. Yuan, L. Guo, L. Yu, "Visual hull construction using adaptive sampling without bounding box," J. Chinese Comput. Syst. 31(9): 1877-1880 (2010).

20. C. H. Esteban, F. Schmitt, "Silhouette and stereo fusion for 3D object modeling," Comput. Vis. Image Und. 96(3): 367-392 (2004).

21. C. Liang, K-Y. K. Wong, "Exact visual hull from marching cubes," Proc. 3rd Int. Conf. on Computer Vision Theory and Applications, Madeira, Portugal, 2: 597-604 (2008).

22. W. X. Cong, G. Wang, D. Kumar et al., "Practical reconstruction method for bioluminescence tomography," Opt. Express. 13(18): 6756-6771 (2005).

23. S. R. Arridge, "Optical tomography in medical imaging," Inverse Probl. 15(2): R41-R93 (1999).

24. S. Osher, M. Burger, D. Goldfarb, J. J. Xu, W. T. Yin, "An iterative regularization method for total variation-based image restoration," Multiscale Model. Simul. 4(2): 460-489 (2005).

25. T. Goldstein, S. Osher, "The Split Bregman method for L1-regularized problems," SIAM J. Imaging Sci. 2(2): 323-343 (2009).

26. M. L. Clapper, H. H. Hensley, W. C. L. Chang, K. Devarajan, M. T. Nguyen, H. S. Cooper, "Detection of colorectal adenomas using a bioactivatable probe specific for matrix metalloproteinase activity," Neoplasia 13(8): 685-691 (2011).

27. S. J. Goldman, E. Chen, R. Taylor, S. Zhang, W. Petrosky, M. Reiss, S. Jin, "Use of the ODD-luciferase transgene for the non-invasive imaging of spontaneous tumors in mice," PloS one 6(3): e18269 (2011).

28. A. Cong, W. Cong, Y. Lu, P. Santago, A. Chatziioannou, G. Wang, "Differential evolution approach for regularized bioluminescence tomography," IEEE T. Bio-Med. Eng. 57(9): 2229-2238 (2010).

29. G. Wang, W. Cong, K. Durairaj, X. Qian, H. Shen, P. Sinn, E. Hoffman, G. McLennan, M. Henry, "In vivo mouse studies with bioluminescence tomography," Opt. Express. 14(17): 7801-7809 (2006).

30. L. Changqing, G. S. Mitchell, S. R. Cherry, "Cerenkov luminescence tomography for smallanimal imaging," Opt. Lett. 35(7): 1109-1111 (2010).

31. Y. Jinzuo, D. Yang, A. Yu, C. Chongwei, T. Jie, "Reconstruction of fluorescence molecular tomography via a nonmonotone spectral projected gradient pursuit method," J. Biomed. Opt. 19(12): 126013-126013 (2014). 